\title{
Decomposition of a Three-Dimensional Discrete Object Surface into Discrete Plane Pieces
}

\author{
Isabelle Sivignon, ${ }^{1}$ Florent Dupont, ${ }^{2}$ and Jean-Marc Chassery ${ }^{1}$
}

\begin{abstract}
This paper deals with the polyhedrization of discrete volumes. The aim is to do a reversible transformation from a discrete volume to a Euclidean polyhedron, i.e. such that the discretization of the Euclidean volume is exactly the initial discrete volume. We propose a new polynomial algorithm to split the surface of any discrete volume into pieces of naive discrete planes with well-defined shape properties, and present a study of the time complexity as well as a study of the influence of the voxel tracking order during the execution of this algorithm.
\end{abstract}

Key Words. Discrete volumes, Digital plane recognition, Surface, Polyhedrization.

1. Introduction. Three-dimensional discrete volumes are used more and more especially in the medical area since they result from MRI and scanners. As two-dimensional images are composed of squares called pixels, these three-dimensional images are composed of cubes called voxels. This structure induces many difficulties in the exploitation and study of these objects: as each cube is stored, the volume of data is very huge, which is a problem in obtaining fluent interactive visualization; the facet structure (voxels's faces) of the discrete object induces many problems to get the nice visualization that is necessary for medicine, as no rendering nor texture algorithm can be applied.

The general idea to solve these problems is to transform discrete volumes into Euclidean polyhedra. Many research activities have already been achieved to find solutions to this problem, using Euclidean geometry or discrete geometry. To get a good visualization of discrete volumes, the method that is most used is the Marching cubes method [1], which considers replacing local voxel configurations by small triangles. Even if this method offers good visualization, it does not provide good data compression (huge number of facets).

Many other research activities have been done in this field, using completely different ideas. The first algorithms dealt with the construction of the convex hull of the considered set of voxels. This study was mainly done by Kim and Rosenfeld who published in [2] a first algorithm to characterize a piece of discrete plane by the convex hull of the discrete surface. This algorithm was then improved by Kim and Stojmenović [3]. This algorithm was not reversible, i.e. the discretization of the Euclidean hull obtained is not the discrete object.

\footnotetext{
${ }^{1}$ Laboratoire LIS, 961 rue de la Houille Blanche, Domaine Universitaire - BP46, 38402 Saint Martin D’Hères Cedex, France. \{sivignon,chassery\} @lis.inpg.fr.

2 Laboratoire LIRIS, 8 Boulevard Niels Bohr, 69622 Villeurbanne Cedex, France. fdupont@liris.cnrs.fr.
}

Received January 21, 2003. Communicated by R. C. Veltkamp.

Online publication October 24, 2003. 
The first reversible algorithm was proposed by Borianne and Françon [4]. In this paper they exposed two methods: one to do a polyhedrization, and another to do the reverse operation, i.e. discretization. For that, they used an approximation by the least-squares method that made it marginal compared with entirely discrete methods.

Another idea was then proposed by Debled [5], [6]. She developed an algorithm to recognize rectangular pieces of naive planes. Then she used this algorithm to decompose the digital surface of symmetric objects (with known symmetries) into pieces of discrete planes. The polyhedrization was not complete here but it was the first approach using discrete plane recognition.

In 1999 Papier [7], [8] presented an algorithm using the Fourier-Motskin algorithm to recognize standard discrete planes on an object surface, each point of the plane being a pointel (vertex of a voxel). The time complexity of this algorithm is high because of the Fourier-Motskin algorithm and, moreover, the polyhedrization done is not reversible.

Finally, in 2000, Burguet and Malgouyres published [9] an approximation algorithm using a curvature computation to choose some germ points and then calculate the skeleton of the discrete surface without those germs (Voronoi diagram). The result is a Delaunay triangulation that approximates and simplifies the original object.

The aim of this paper is to present the first steps to achieve a totally discrete and reversible polyhedrization. We use discrete geometry that seems to fit best the structure of the processed objects. Reversibility means that from a discrete object, we can get a Euclidean polyhedron whose digitalization is exactly the former discrete volume. This property enables many applications and we give two of them here. First, this can lead to efficient data compression describing the volume by the set of all the faces of the Euclidean polyhedron: no loss of data and no loss of information in the compressed object. After this transformation, we can apply morphological operations on the reconstructed Euclidean polyhedron and then retrieve the discrete volume obtained after these operations.

In the next section, we give the basic definitions of discrete geometry. Then we present in detail the naive plane recognition algorithm that we use in the following, giving some improvements and new properties. In Section 4, after a short state of the art, we expose our segmentation algorithm. Section 5 deals with the algorithm's time complexity. Then, in the next section, we propose a study of the voxel processing order and its influence on the final surface decomposition. Before a few words of conclusion, we finally present some performance and image results on generated and real volumes.

2. Basic Definitions and Properties. In this first part we focus in a few words on the basic objects and definitions of discrete geometry. All the following definitions lie in a discrete three-dimensional space. This space is defined as a unit cubic mesh centered on points having integer coordinates. The vertices of each cell (cube) of the mesh correspond to points with half-integer coordinates.

A voxel or $\mathbb{Z}^{3}$ point or discrete point is assimilated with the unit closed cube of the mesh. Then voxel coordinates are the coordinates of the corresponding cube center. Faces, edges and vertices of a voxel are respectively called surfels, linels and pointels.

In $\mathbb{Z}^{3}$, three voxel neighborhoods (Figure 1) are classically used. They are defined with the two distances called the Manhattan distance, denoted $d_{6}$, and the Chessboard 


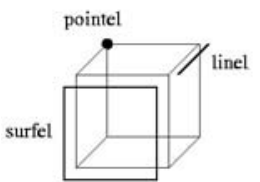

(a)

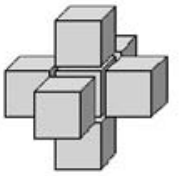

(b)

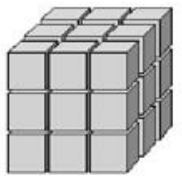

(c)

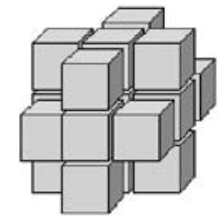

(d)

Fig. 1. A voxel and the three classical neighborhoods.

distance, denoted $d_{26}$ :

$$
\begin{aligned}
d_{6}(M, P) & =\left|x_{m}-x_{p}\right|+\left|y_{m}-y_{p}\right|+\left|z_{m}-z_{p}\right|, \\
d_{26}(M, P) & =\max \left(\left|x_{m}-x_{p}\right|,\left|y_{m}-y_{p}\right|,\left|z_{m}-z_{p}\right|\right) .
\end{aligned}
$$

Two voxels $M$ and $P$ are 6-neighbors $(6-\mathrm{N})$ if and only if $d_{6}(M, P) \leq 1 . M$ and $P$ are 26-neighbors (26-N) if and only if $d_{26}(M, P) \leq 1$. In other words, two points are 6-N if they have a common face, $26-\mathrm{N}$ if they have a common face, a common edge or a common vertex. This point of view suggests another neighborhood for the case of two voxels sharing a common face or a common edge, called 18-N $\left(d_{6}(M, P) \leq 2\right)$.

A classical way to define a discrete line or a discrete plane is to consider the digitization of a Euclidean line or plane on a unit grid with a given digitization scheme. However, as in Euclidean space, there exist arithmetical definitions of discrete planes and lines. Those definitions were given by Reveillès [10] and then generalized to hyperplanes by Andrès [11].

A digital plane (Figure 2) of normal vector $(a, b, c)$, translation parameter $r$ and arithmetical thickness $\omega \in \mathbb{N}$ is defined as the set of points $M(x, y, z) \in \mathbb{Z}$ satisfying the double inequality:

$$
0 \leq a x+b y+c z+r<\omega,
$$

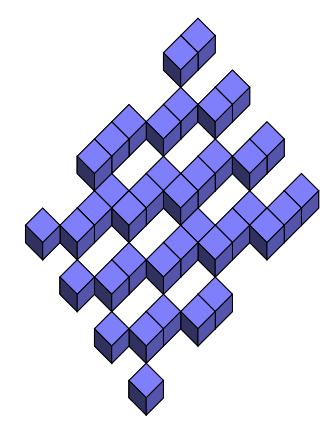

(a)

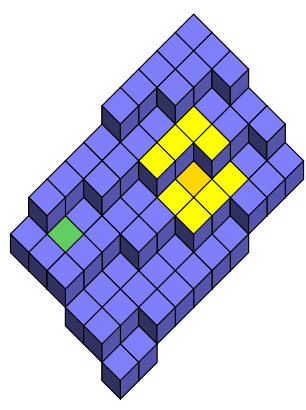

(b)

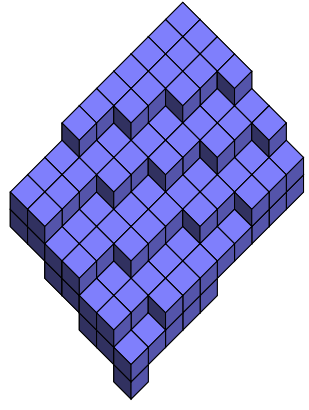

(c)

Fig. 2. A discrete plane: $0 \leq 6 x+13 y+27 z<\omega$ with different thicknesses: (a) $\omega=15$, a thin plane with holes; (b) $\omega=27$, a naive plane; (c) $\omega=46$, a standard plane. A tricube is also depicted onto the naive plane. 
where $a, b, c$ are not all null and satisfy $\operatorname{gcd}(a, b, c)=1$. A discrete plane such that $\omega=|a|+|b|+|c|$ is called standard. A discrete plane such that $\omega=\max (|a|,|b|,|c|)$ is called naive (see Figure 2 for an example).

The thickness parameter determines the connectivity of the plane. In fact, naive planes are the thinnest 18-connected planes without 6-holes and therefore they are very well adapted for object surface study. In the rest of the paper we deal with naive planes, denoted $P(a, b, c, r)$.

Finally, naive discrete plane can be decomposed into primitive elements called tricubes: the tricube at point $(i, j)$ of the naive plane $P(a, b, c)$ with $|a| \leq|b| \leq|c|$ is defined as the set $\{(x, y, z) \in P \mid i \leq x \leq i+3, j \leq y \leq j+3\}$.

3. Recognition of a Piece of Discrete Naive Plane. We present in this part an algorithm proposed by Vittone and Chassery [12] to recognize digital plane segments. Some new properties are also proved.

3.1. Description of the Algorithm. Given a Euclidean plane $P$ defined by $a x+b y+$ $c z+r=0$, where $0 \leq a \leq b \leq c$ and $c \neq 0$, the $O B Q$ (Object Boundary Quantization) discretization of $P$ is the set of all points $M(x, y, z)$ of the mesh on or "under" $P$. For $x, y \in \mathbb{Z}$, this method consists of rounding $z$ to the lower integer value. The result of such a discretization is the naive plane with parameters $(a, b, c, r)$.

In [13] and [12] Vittone presents an algorithm that solves in polynomial time the following problem (the so-called recognition problem):

Let $S$ be a set of voxels containing the origin $(0,0,0)$ and let $n$ be other voxels $\left(i_{q}, j_{q}, k_{q}\right), q=1, \ldots, n$. What is the set $\bar{S}$ of the parameters $(\alpha, \beta, \gamma) \in \mathbb{R}$ with $0 \leq \alpha \leq \beta<1$ and $0 \leq \gamma \leq 1$ such that all the voxels of $S$ belong to the OBQ discretization of $P: \alpha x+\beta y+z+\gamma=0$ ?

Then we look for the set $\bar{S}$ defined by

$\bar{S}=\left\{(\alpha, \beta, \gamma) \in\left[0,1\left[^{2} \times[0,1], \alpha \leq \beta \mid \forall(x, y, z) \in S, \quad 0 \leq \alpha x+\beta y+z+\gamma<1\right\}\right.\right.$.

We consider the duality of the double inequality of the former formula. Indeed, let $P$ be a Euclidean plane defined by $z=-(\alpha x+\beta y+\gamma)$. This equation represents all the points $(x, y, z)$ belonging to $P$. We rewrite the equation as $\gamma=-(x \alpha+y \beta+z)$. Then, in the dual space $(0, \alpha, \beta, \gamma)$ (also called the parameter space), this equation represents all the planes containing the point $(x, y, z)$. In this space a plane $(a, b, c, r)$ is the point $(a / c, b / c, r / c)$ if $c=\max (a, b, c)$.

Since each voxel generates a double inequality, in the dual space each voxel of $S$ is represented by a half-open strip delimited by two parallel planes. For a given voxel $(x, y, z)$, this area represents the set of Euclidean planes parameters whose OBQ discretization contains the voxel $(x, y, z)$. Finally, $\bar{S}$ is the intersection in the dual space of $n$ half-opened strips delimited by two Euclidean planes $P\left(i_{q}, j_{q}, k_{q}\right)$ and $P\left(i_{q}, j_{q}, k_{q}-1\right)$, $q=1, \ldots, n$.

This is the main point of the recognition algorithm: each voxel constrains the solution area in the dual space with a half-opened strip. The intersection of those half-spaces can be found step by step by adding one voxel after the other. At the end, $\bar{S}$ can be 


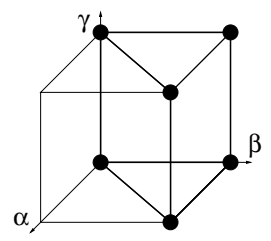

Fig. 3. The initial set of solutions.

a polyhedron, a polygon, a line segment or empty. In the last case the voxels are not coplanar.

We present here a sketch of the final algorithm. Let $M(x, y, z)$ be a voxel and let $S$ be the set containing $M$ and $p$ other voxels with coordinates $\left(x+i_{q}, y+j_{q}, z+k_{q}\right), q=$ $1, \ldots, p$. The aim is to find the set of the naive planes containing all the $p+1$ voxels of $S$, $M$ being the origin. The computation of the half-spaces intersection returns the solution area $\bar{S}$ and the final solutions are, after translation, the planes $P(a, b, c, r-(a x+b y+c z))$ such that $(a / c, b / c, r / c)$ is in $\bar{S}$.

Since $0 \leq \alpha \leq \beta<1$ and $0 \leq \gamma \leq 1$, the initial solution area is delimited by the projections of the six vertices of

$$
B_{0}=\{(0,0,0,1),(0,1,0,1),(1,1,0,1),(0,0,1,1),(0,1,1,1),(1,1,1,1)\}
$$

(Figure 3) onto the dual space. In the rest of this paper, $B_{q}$ stands for the set of points in $\mathbb{N}^{4}$ such that their projections in the parameter space are the vertices of the solution area for the first $q$ voxels. Hence, $\bar{S}$ is the projection of translated $B_{p+1}$ in the parameter space.

We denote $L_{q}(a, b, c, r)=a i_{q}+b j_{q}+c k_{q}+r$ and $L_{q}^{+}(a, b, c, r)=L_{q}(a, b, c, r)-c$. Let $(a, b, c, r)$ be the normal vector of a plane $P$ solution after step $q$. Then, at step $q+1$, this plane is still a solution if and only if $L_{q+1}(a, b, c, r)$ and $L_{q+1}^{+}(a, b, c, r)$ have opposite signs, i.e. in the dual space, the point corresponding to the plane $P$ is between the two planes defined by the voxel $\left(i_{q+1}, j_{q+1}, k_{q+1}\right)$.

The following algorithm takes as input a voxel $V\left(i_{q}, j_{q}, k_{q}\right)$ and the set $B_{q-1}$ solution for the first $q-1$ voxels, and computes the set $B_{q}$ of the solution polyhedron vertices after the addition of $V$.

Function Add_voxel $\left(B_{q-1}, V\right)$

Initialization. $B_{q}=\emptyset$.

$L_{q}(a, b, c, r)=a i_{q}+b j_{q}+c k_{q}+r$ and $L_{q}^{+}(a, b, c, r)=L_{q}(a, b, c, r)-c$.

\section{Main loop.}

(1) For all $V_{1}$ belonging to $B_{q-1}$ do

$$
\begin{aligned}
& \text { If } L_{q}\left(V_{1}\right)=0 \text { or } L_{q}^{+}\left(V_{1}\right)=0 \text { then put } V_{1} \text { in } B_{q} \\
& \text { Else if } L_{q}\left(V_{1}\right)>0 \text { and } L_{q}^{+}\left(V_{1}\right)<0 \text { then put } V_{1} \text { in } B_{q}
\end{aligned}
$$

$$
\text { Else }
$$


For all $V_{2}$ in $B_{q-1}, V_{2} \neq V_{1}$, such that $L_{q}\left(V_{1}\right)$ and $L_{q}\left(V_{2}\right)$ or $L_{q}^{+}\left(V_{1}\right)$ and $L_{q}^{+}\left(V_{2}\right)$ have opposite signs

- Compute the intersection $I$ of the line $\left(V_{1} V_{2}\right)$ and the plane $L_{q}(X)=0$ (or $L_{q}^{+}(X)=0$ )

end for

end for

\section{Result. Return $B_{q}$.}

The result of this function is the set of the solution polyhedron vertices after the processing of $q$ first voxels. Hence, to check if a set of voxels $S$ are coplanar, it is enough to call the function Add_voxel for one voxel after the other, each time using the last $B_{q}$ computed. In the rest of this paper we call the algorithm that recognizes a piece of the plane the recognition algorithm.

3.2. Properties and Improvements. This polyhedron $\bar{S}$ is the intersection of half-open strips. Hence, although the points that are linearly dependent with positive weights to the vertices of $\bar{S}$ are necessarily solutions, this algorithm does not make precise whether the vertices, edges and faces of $\bar{S}$ are solutions or not.

PROPOSITION 1. Let $S=\left\{\left(i_{q}, j_{q}, k_{q}\right), q=1, \ldots, p\right\}$ be a set of $p$ voxels, and let $\bar{S}$ be the solution polyhedron obtained with the recognition algorithm. If $\bar{S}$ is not empty, let $N=\left\{N_{i}, i=1, \ldots, m\right\}$ be the set of vertices of $\bar{S}$. Then $N_{i}$ is a solution if and only if $\forall q, 1 \leq q \leq p, L_{q}^{+}\left(N_{i}\right) \neq 0$.

Let $E$ be a point of the edge $\left(N_{i}, N_{j}\right)$. If $N_{i}$ or $N_{j}$ is a solution, then $E$ is also a solution.

ProOf. Let $N_{i}(a, b, c, r)$ be a vertex of $\bar{S}$. Suppose that there exists a voxel $\left(i_{q}, j_{q}, k_{q}\right)$ such that $L_{q}^{+}\left(N_{i}\right)=0$. This means that $N_{i}$ belongs to the plane $\left(i_{q}, j_{q}, k_{q}-1\right)$ in the dual space. Since this plane is the open limit of the solution area, $N_{i}$ is not a solution. On the other hand, suppose that $N_{i}$ is not a solution, and show that there exists a voxel $\left(i_{q}, j_{q}, k_{q}\right)$ such that $L_{q}^{+}\left(N_{i}\right)=0$. By construction, two kinds of non-solution points exist: those that are not in the solution polyhedron, and those that belong to an open side of the polyhedron. As $N_{i}$ is a non-solution vertex of the solution polyhedron, it belongs to a plane that is an open side of the polyhedron, i.e. a plane whose normal vector is $\left(i_{q}, j_{q}, k_{q}-1\right)$. Then there exists $\left(i_{1}, j_{q}, k_{q}\right)$ such that $a i_{q}+b j_{q}+c\left(k_{q}-1\right)+r=0$, and then $L_{q}^{+}\left(N_{i}\right)=0$.

Let $E$ be a point of the edge $\left(N_{i}, N_{j}\right)$ with solution $N_{i}$. Suppose that $E$ is not a solution. Then there exists a half-open strip that does not contain $E$. As $E$ is on an edge of the polyhedron, $E$ belongs to the open plane of a strip. Either this plane contains the edge $\left(N_{i}, N_{j}\right)$ and then this leads to a contradiction, or this plane cuts this edge in $E$, and then one of the two vertices $N_{i}$ or $N_{j}$ is outside the strip. If $N_{i}$ is outside, then we get the contradiction. Otherwise, if $N_{i}$ is a solution, then $N_{j}$ is not. As $E$ is on the edge $\left(N_{i}, N_{j}\right), N_{j}$ does not belong to the open plane, which implies that $N_{j}$ is not a vertex of $\bar{S}$. Contradiction. 
COROLlaRY 1. Let $E$ be a point of a face $F$ of $\bar{S}$. Let $N_{i}, i=1, \ldots, n, n \geqq 2$, be the set of vertices of $F$. If at least one $N_{i}$ is a solution and if $E$ is not on an edge of the face, then $E$ is also a solution.

PROOF. For $n=2$, see Proposition 1. For $n>2$, the demonstration is nearly the same. Suppose that $E$ is not a solution. As $E$ is on a face of the polyhedron, $E$ belongs to one of the open planes of the strips. If this plane contains the face $F$, then we get the contradiction as $N_{i}$ belongs to this face. Otherwise, there exists an open plane containing $E$. As $E$ is not on an edge and as $\bar{S}$ is convex, this plane cuts the face $F$ in at least two edge points. This plane splits the space into two half-spaces, one containing points that do not belong to $\bar{S}$. Therefore, at least one vertex of $F$ will be in this half-space, a contradiction.

Now we focus on line (6) of the function Add_voxel presented in Section 3.1. Many efficient algorithms exist to compute the intersection of a polyhedron and a plane (see for instance Chapter 7 of [14]). Those algorithms return the set of vertices of the polyhedron as rational numbers. However, to get the plane normal vectors corresponding to the vertices coordinates, we must have those coordinates in fractional form. Instead of computing the polyhedron first and then transforming each vertex coordinate, it is better to compute them directly as fractions.

In [13] that was done using a modified version of Grabiner's algorithm [15]. This algorithm uses Farey series and their properties to compute the new vertices $v$ with a dichotomy method. The time complexity is then $\mathcal{O}(\log (n))$ if $v$ is between two vertices $v_{1}$ and $v_{2}$ such that $d\left(v_{1}, v_{2}\right)=n$ where $d$ denotes the Euclidean distance. We propose here to compute directly those coordinates keeping the value of numerators and denominators at each step of the computation. This step can be done in $\mathcal{O}(1)$ time with the following algorithm.

$V_{1}$ and $V_{2}$ are two vertices of the current solution polyhedron and $P$ is a plane in the dual space. This algorithm will compute the parameters of the Euclidean plane whose representation in the dual space is the intersection point between the line $\left(V_{1}, V_{2}\right)$ and the plane $P$.

\section{Function Plane_line $\left(V_{1}, V_{2}, P\right)$}

Initialization. $V_{1}\left(a_{1}, b_{1}, c_{1}, r_{1}\right), V_{2}\left(a_{2}, b_{2}, c_{2}, r_{2}\right), P: \alpha i+\beta j+k+\gamma=0$, in the dual space $(0, \alpha, \beta, \gamma)$.

Let $p$ be the intersection point of the line $\left(V_{1}, V_{2}\right)$ and the plane $P$.

\section{Computation.}

Compute $N=-i a_{1} c_{2}-j b_{1} c_{2}-r_{1} c_{2}-k c_{1} c_{2}$.

Compute $D=i\left(a_{2} c_{1}-a_{1} c_{2}\right)+j\left(b_{2} c_{1}-b_{1} c_{2}\right)+\left(r_{2} c_{1}-r_{1} c_{2}\right)$.

Result. The three coordinates have a common denominator: $p_{d}=N \times c_{1} c_{2}$. The three numerators are $p_{n}=\left(N\left(a_{2} c_{1}-a_{1} c_{2}\right)+a_{1} c_{2} D, N\left(b_{2} c_{1}-b_{1} c_{2}\right)+\right.$ $\left.b_{1} c_{2} D, N\left(r_{2} c_{1}-r_{1} c_{2}\right)+r_{1} c_{2} D\right)$.

It is easy to retrieve the coordinates of the corresponding plane with the definition of the dual space: for instance, if $|c|=\max (|a|,|b|,|c|)$, the plane coordinates 
are $\left(N\left(a_{2} c_{1}-a_{1} c_{2}\right)+a_{1} c_{2} D, \quad N\left(b_{2} c_{1}-b_{1} c_{2}\right)+b_{1} c_{2} D, \quad p_{d}, \quad N\left(r_{2} c_{1}-r_{1} c_{2}\right)\right.$ $\left.+r_{1} c_{2} D\right)$.

To conclude this part, this recognition algorithm offers some properties that are useful for the next step, i.e. applying this algorithm on a discrete surface:

- it recognizes a naive discrete plane: the minimal thickness of these planes implies that the object surface is enough to do a recognition, we do not need interior voxels;

- it is incremental: the voxels can be added one by one;

- for a given set of voxels, the adding order does not have an influence on the final result;

- it returns the set of vertices of the solution polyhedron: so, we have the complete set of the solution planes normal vectors.

4. General Algorithm. Recognizing discrete planes is the first step of a most general goal: the polyhedrization of a discrete object. This section describes a new algorithm that splits the discrete surface of an object into naive plane pieces. We will also see that this algorithm has features which make it especially well adapted to get a totally discrete and reversible polyhedrization.

We consider 18-connected objects with a 6-connected background. In the rest of the paper we call the set of surfels that belong simultaneously to an object voxel and to a background voxel the surface. In other words, the surface is the set of visible surfels. As each voxel has six faces, those six faces define six directions that we consider symmetrically during the algorithm's description.

\section{Algorithm Decompose-discrete-surface}

Initialization. For each object voxel, locate the surface surfels, $S$.

Initialize the number of planes cpt to -1 .

Initialize the list To-process with the empty list.

Let $B$ be a set of vertices of a solution polyhedron: $B_{0}$, the initial set, depends on the current direction.

\section{Main loop.}

(1) For each object direction $d$

(2) For each object voxel $V$

(3) Let $s_{0}$ be the surfel of $V$ in the direction $d$;

If $s_{0} \in S$ and $s_{0}$ has never been treated then

$$
\text { origin }=s_{0} \text {; }
$$$$
c p t=c p t+1 \text {; }
$$$$
\text { put } s_{0} \text { in To-process; }
$$$$
B=B_{0} \text {; }
$$

While To-process is not empty

choose one surfel $s$ in To-process;

$B_{\text {save }}=B$;

For each of the 8-neighbors $s_{n}$ of $s$

$$
B=\operatorname{Add} \_\operatorname{voxel}\left(B, s_{n}\right)
$$




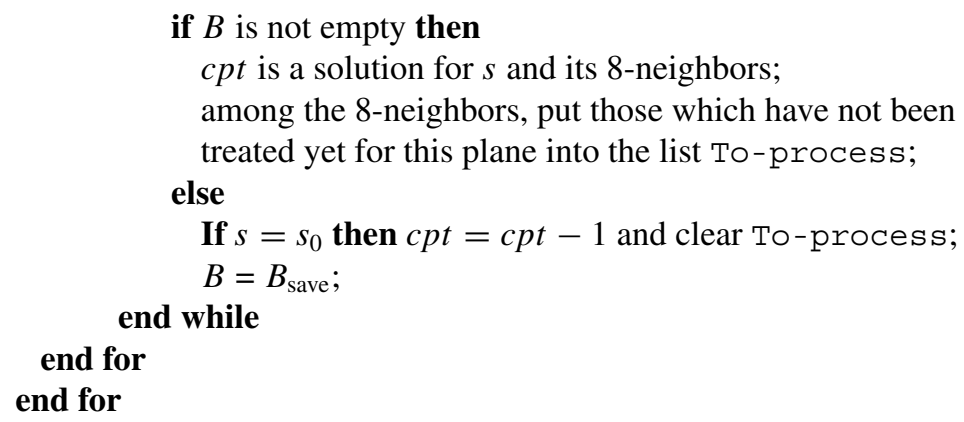

Result. For each surfel: a list of all the plane numbers it belongs to.

For each piece of plane: the set of all the solution polyhedron vertices.

In this algorithm the solution polyhedron is represented by the set of its vertices denoted by $B$. Each time the function Add_voxel is called, the set $B$ is modified. We save the value of $B$ before the addition of the 8-neighbors of a given surfel $s$. So, if $s$ is not a tricube center, we can recover the solution polyhedron as it was before the processing of $s$ 's neighbors (lines (18) and (19)).

During the execution, for each surfel we create a list containing all the plane numbers to which this surfel belongs. Moreover, at the end of each piece of plane recognition, we keep in an appropriate structure the coordinates of the solution polyhedron vertices.

We analyze the properties of this algorithm:

- During the processing of a surfel, either eight faces are added to the current plane or zero: indeed, if a surfel is a tricube center, then we add all of them to the current plane, otherwise, none of them are added (even those which could belong to the plane). This implies that every surfel of a recognized naive plane has a least three neighbors belonging to this plane. Indeed, a face that belongs to a piece of plane must have a neighbor that is a tricube center. Hence, only two cases are possible (see Figure 4). As a consequence, recognized regions have a "regular form".

- A surfel can belong to many pieces of planes: indeed, no restrictions nor choices are done during the expansion of the planes. Then naive planes are extended to their maximum under the constraint given before.

The second property can be seen as an advantage or as a problem. Indeed, if we do not allow discrete plane covering, the limit between two planes is easy to handle. However, we can get many very small pieces of plane at the end of the algorithm and, hence, allowing plane covering reduces the influence of the seeds used for the pieces of planes.
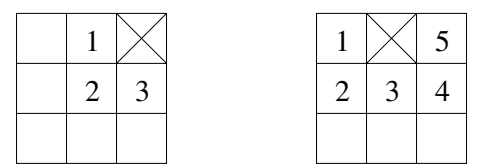

Fig. 4. A surfel of a piece of plane has at least three neighbors in this plane. 
Moreover, to get a reversible polyhedrization, the border of a piece of plane should be a discrete line. Without covering, we have no means to control the border of the pieces of plane.

5. Time Complexity. In this section we give a polynomial bound on the algorithm's time complexity. This study is split into two parts: first, the time complexity of the function Add_voxel presented in Section 3; then the time complexity of the algorithm Decompose-discrete-surface described in Section 4.

5.1. Add_voxel Time Complexity. The first loop of this algorithm covers the elements of the set $B_{q}$. To bound the cardinality of this set, we have to bound the number of vertices of a polyhedron according to its number of faces. This is a classical result in computational geometry (see Chapter 7 of [14] for instance) that we recall here:

THEOREM 1. Let $P$ be a convex polyhedron with $n$ faces. Then $P$ has at most $2 n-4$ vertices.

In the algorithm, $B_{0}$ is a polyhedron with five faces. As the addition of one voxel is equivalent to the addition of two parallel planes in the dual space, after step $q$, the solution polyhedron has at most $2(2 q+5)-4=4 q+6$ vertices. As a matter of fact, the first loop of the function Add_voxel is done in $\mathcal{O}(q)$ time where $q$ is the number of voxels of the piece of plane. Nevertheless, in practice, the number of vertices of $B_{q}$ is much smaller than $q$.

In the loop the first two tests can be done in constant time. The second loop does a new cover of the set $B_{q}$ and is carried out in $\mathcal{O}(q)$ time. For the computation of the plane/line intersection, we saw that here we need to keep some particular knowledge on the values found for the intersection point, and we proposed in Section 3.2 an algorithm that solves this problem in constant time. To recover the parameters of the solution planes, we will need after this algorithm a step to normalize the parameters (using Euclid's algorithm for instance to compute the gcd of the three denominators). This normalization can be done either for each $B_{q}$, or only at the end, for the vertices of $\bar{S}$.

For the function Add_voxel, we finally find an $\mathcal{O}\left(q^{2}\right)$ time complexity, where $q$ is the number of voxels of the piece of plane.

5.2. Decompose-discrete-surface Time Complexity. We analyze line by line how this algorithm runs. Let $n$ be the number of voxels which have a surfel belonging to the object surface. As a voxel has six faces, the first loop (line (1)) is done exactly six times. The second loop (line (2)) is run $n$ times as we have $n$ surface voxels. All the tests and instructions done between lines (3) and (8) run in constant time.

The time complexity of the loop line (9) depends on the maximum number of elements in To-Process.

PROPOSITION 2. At step number $q$ (after q first voxels) the maximum number of elements in To-Process is $4 q+4$. 
PROOF. After processing the first surfel, we put its 8-neighbors in To-Process. Moreover, we have seen in Section 4 that any surfel belonging to a piece of plane has at least three neighbors in this plane. This means that at any time during the algorithm, each surfel of To-Process has at least two neighbors in this list. During the treatment of one surfel of the list, we delete this element from the list and we add its 8-neighbors. However, since at least three of them are already in the list, we add at most five for its neighbors. Finally, we add at most $5-1=4$ surfels at each step. Hence, at step number $q$, this list has at most $8+4(q-1)=4 q+4$ elements.

So, for the recognition of a naive plane with $q$ voxels, this loop will be done at most $4 q+4$ times. The choice in line (10) can be done in constant time, and in line (11), saving $B$ needs a cover of the set $B$, which is done in $\mathcal{O}(q)$ time for a plane with $q$ voxels. Moreover, for a naive plane with $q$ voxels, the function Add_voxel runs in $\mathcal{O}\left(q^{2}\right)$ time, and the loop line (12) in $\mathcal{O}\left(8 q^{2}\right)=\mathcal{O}\left(q^{2}\right)$ time. All the tests and instructions done between lines (14) and (18) run in constant time. The restitution of $B$, line (19), is done

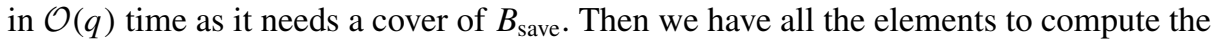
global time complexity of this algorithm as a function of $n$, the number of voxels which have a surface surfel, and $p$, the size of the biggest recognized piece of plane. We get

$$
6 n \times p \times\left(2 p+8 p^{2}\right),
$$

which leads to a final time complexity of $\mathcal{O}\left(n p^{3}\right)$.

6. Study on the Voxel Processing Order. During execution of the algorithm Decompose-discrete-surface, many choices have to be made concerning the order in which to process the voxels. They have an influence on the final decomposition we get: a given set of choices induces a different decomposition. Therefore, a study is useful to know if there exists a strategy leading to a "better" decomposition. In this section we study this influence, comparing the results obtained with different strategies.

In the algorithm, three main choices are made for the tracking order. Indeed, in lines (1), (2), (10) and (12), no details are given concerning the processing order for these different steps. However, we can easily see that the choice made in line (10) does not influence the result: since our approach is surfel based, the recognition done for one direction has no influence on the recognitions done for the others. Then three choices remain:

- the origin of each piece of plane (line (2));

- the next voxel to process during the recognition of a piece of plane (line (10));

- the tracking order of the 8-neighbors of a given voxel which determines the structure of the list To-Process (line (16)).

In this study we give an insight on the influence of the last two.

First, we can notice that the order in which we process the 8-neighbors of a given voxel determines the order in which those neighbors are inserted into the list To-Process. Hence, the planes growing shape depends on two inter-dependent choices.

In the following we present various strategies defined from those two choices. 


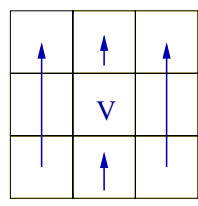

(a)

\begin{tabular}{|c|c|c|c|c|}
\hline 14 & 15 & 16 & 18 & 23 \\
\hline 13 & 2 & 4 & 7 & 22 \\
\hline 10 & 1 & $\mathrm{~V} 0$ & 6 & 21 \\
\hline 9 & 0 & 3 & 5 & 20 \\
\hline 8 & 11 & 12 & 17 & 19 \\
\hline
\end{tabular}

(b)

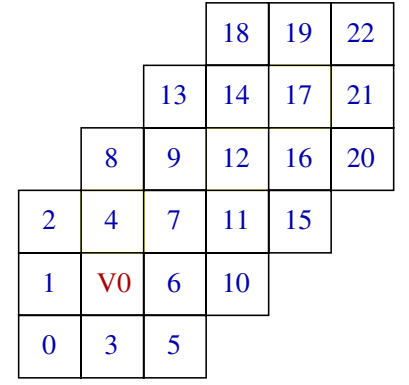

(c)

Fig. 5. Strategy 1: (a) the 8-neighbors tracking; (b) propagation with the first element of the list To-Process; (c) propagation with the last element.

6.1. Different Strategies. The first strategy is also the simplest one to implement. In Figure 5 we present first the 8-neighbors tracking and then the propagation scheme depending on which surfel we choose in the list of surfels To-Process. The numbers on the surfels refer to the order in which they are added in the list To-Process. With this first order, taking the last element of the list at each step leads to a very linear propagation scheme. This induces a main direction for the planes propagation. In fact, for any neighborhood tracking, choosing the last element of the list leads to a main direction given by the position of the last element processed during the 8-neighborhood tracking. If we take the first element of the list as a following surfel, we get the propagation drawn in Figure 5. With this tracking, the left-down corner is always treated before the other sides, and the expansion is not regular nor isotropic.

Figure 6 illustrates a second strategy. The 8-neighbors tracking is now a clockwise tracking around the processed voxel (any other tracking around the voxel gives symmetrical results). The propagation obtained with the choice of the first surfel of the list is more isotropic than the previous one, even if the left-down corner is still processed first in an irregular way when we get further from the plane origin.

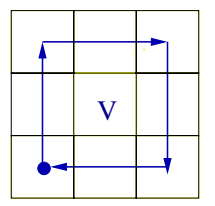

(a)

\begin{tabular}{|c|c|c|c|c|c|c|}
\hline 34 & 35 & 36 & 37 & 38 & 39 & 40 \\
\hline 33 & 14 & 15 & 16 & 17 & 18 & 41 \\
\hline 30 & 13 & 2 & 3 & 4 & 19 & \\
\hline 29 & 10 & 1 & V0 & 5 & 20 & \\
\hline 26 & 9 & 0 & 7 & 6 & 21 & \\
\hline 25 & 8 & 12 & 11 & 23 & 22 & \\
\hline 24 & 28 & 27 & 32 & 31 & & \\
\hline
\end{tabular}

(b)

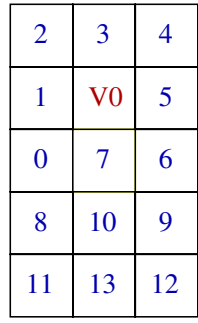

(c)

Fig. 6. Strategy 2: (a) the 8-neighbors tracking; (b) propagation with the first element of the list To-Process; (c) propagation with the last element. 


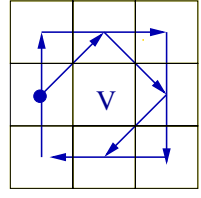

(a)

\begin{tabular}{|l|l|l|l|l|l|l|}
\cline { 2 - 6 } \multicolumn{1}{c|}{} & 32 & 30 & 29 & 31 & 33 & \multicolumn{1}{|c|}{} \\
\hline 28 & 21 & 12 & 11 & 13 & 22 & 37 \\
\hline 26 & 10 & 5 & 1 & 6 & 15 & 35 \\
\hline 24 & 8 & 0 & $\mathrm{~V} 0$ & 2 & 14 & 34 \\
\hline 25 & 9 & 4 & 3 & 7 & 16 & 36 \\
\hline 27 & 20 & 18 & 17 & 19 & 23 & 38 \\
\hline & 42 & 40 & 39 & 41 & 43 & \multicolumn{2}{|c}{} \\
\cline { 2 - 6 } & & &
\end{tabular}

(b)

Fig. 7. Strategy 3: (a) the 8-neighbors tracking; (b) propagation with the first element of the list To-Process.

The main problem with those two strategies is that it is difficult to handle exactly the propagation even close to the origin.

A third method is illustrated in Figure 7. This 8-neighbors tracking processes the voxels that are closer to the origin of the piece of plane first: the four 4-neighbors are first processed, and then the four 8-neighbors. As we saw that choosing the last element of the list induces linear propagations, we just show here the propagation obtained with the choice of the first element. We see that even after a big number of steps, the propagation scheme is always the same: the four directions ("sides") are processed one after the other in the clockwise direction. During the processing of one side, the surfels are processed according to their distance to the origin. After processing the four sides, the four corners are treated. So, the propagation is perfectly defined in this case, and is isotropic as each direction is processed in the same way as another, even if one direction is processed first.

6.2. Comparison Results. In the following we give some results for the comparison of the three tracking orders presented previously. To do so, we use the following criterion and objects: since a sphere is a symmetric object in all the directions, it would be nice to get pieces of planes that have nearly the same size. Hence, for a sphere, the standard deviation/average for the size of the recognized pieces of planes should be as small as possible.

In the rest of this section we denote order 1 (resp. 2,3) to be the one which corresponds to the first (resp. second, third) strategy on the previous section, independently of the choice of the next voxel to process.

Figures 8 and 9 present the two comparisons we propose. The curves depicted are spline approximations of the discrete results.

In the first comparison (Figure 8), each diagram represents the curves for one given tracking order, and each curve is the result choosing the first or the last voxel of the list. For all the strategies, the general shape of the curves is chaotic. This is due to the discrete nature of the data. Nevertheless, the curves have similar behaviors: for instance, all the curves have a local maximum when the radius is 5 or 8 . It is quite easy to see that on those three first graphs, the curve corresponding to the choice of the last voxel of the list 


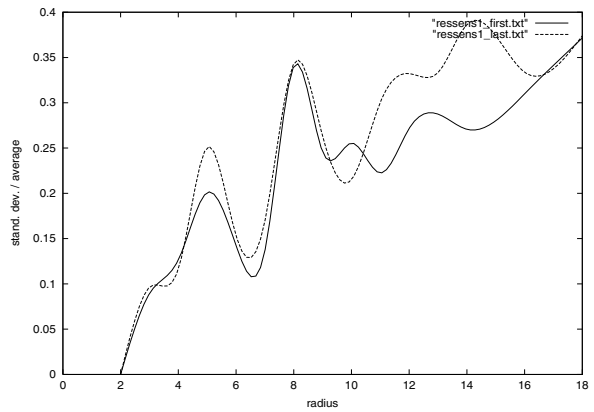

(a)

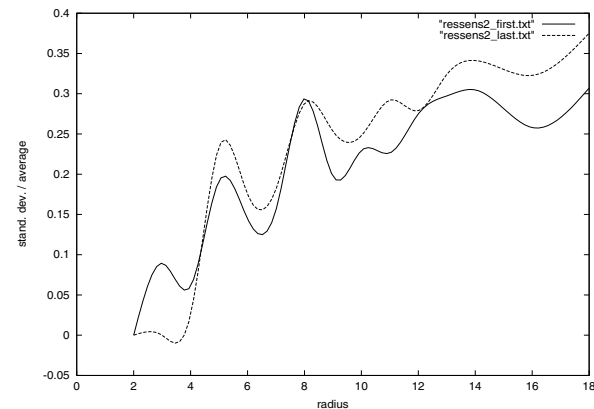

(b)

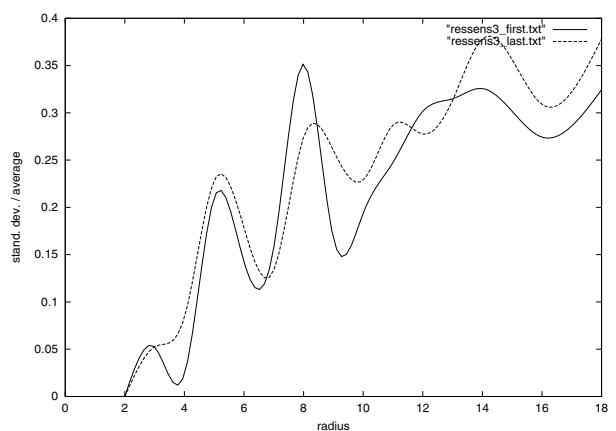

(c)

Fig. 8. Comparison for the choice of the next voxel to process: (a) order 1; (b) order 2; (c) order 3.

is globally worse than the one corresponding to the first voxel of the list. This suggests that the more isotropic the growing shape is, the better the result is.

The results of the second comparison are depicted in Figure 9. In this case the first voxel of the list is chosen and the comparison is done over the three different orders. This figure shows that the three curves cross over, keeping very close values for any sphere radius. Hence, we cannot deduce from this graph that one tracking order is better

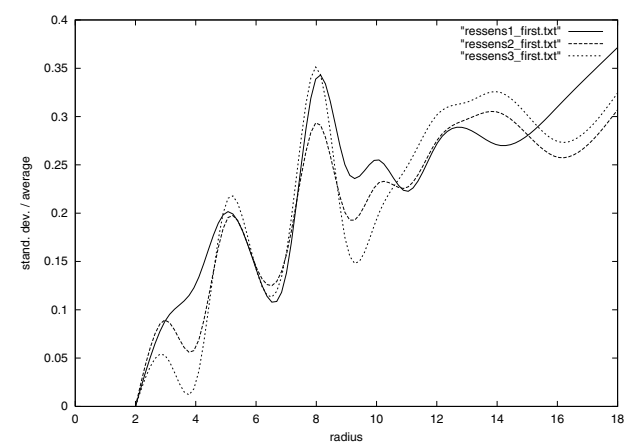

Fig. 9. Comparison for the 8-neighbors tracking order. 
than another one, and according to the very similar values we obtain, this suggests that those three orders have nearly the same behavior. Finally, it seems that the tracking order chosen does not have a very big influence on the resulting quality provided that it does not lead to a linear growing shape.

Nevertheless, it would be interesting to see if the global behavior becomes stable when the radius of the sphere increases up to huge values, i.e. if one tracking order becomes better than the others, or if the curves always cross whatever the radius is. The treatment of very huge objects leads to implementation problems: indeed, since we work with integer fractions in the dual space, we quickly get some very long integers. The solution is to use a library to handle integers with infinite precision and this work is now in progress.

7. Results. In this section we present some results about speed performances and images resulting from our algorithm.

7.1. Performance Results. We did some tests for performance results on a Linux OS with a $1.8 \mathrm{GHz}$ processor. The algorithm is implemented in $\mathrm{C}++$ with no particular optimizations. Figure 10(a) shows the results obtained for cubes of different sizes. In this figure we use logarithmic scales for the two axes. Hence, if the processing time depends directly on a power of the size of the object, then the graph is a straight line. Moreover we only consider the time spent for the recognition of the pieces of planes, not including the input/output and display operations.

As the tracking order does not influence the result for a cube (the six faces are always found), we chose the tracking order that minimizes the lists tracking in the algorithm, i.e. the first order with the choice of the last element of the list To-Process. We can moreover notice that even if choosing the first element of the list To-Process induces one more list tracking in the time complexity computation, in practice, this choice has no effect on performance results.

Using Section 5 results, we can evaluate the time complexity for a cube of side $n$ : the number of surface surfels is in $\mathcal{O}\left(n^{2}\right)$ and the size of the biggest plane is in $\mathcal{O}\left(n^{2}\right)$ too,

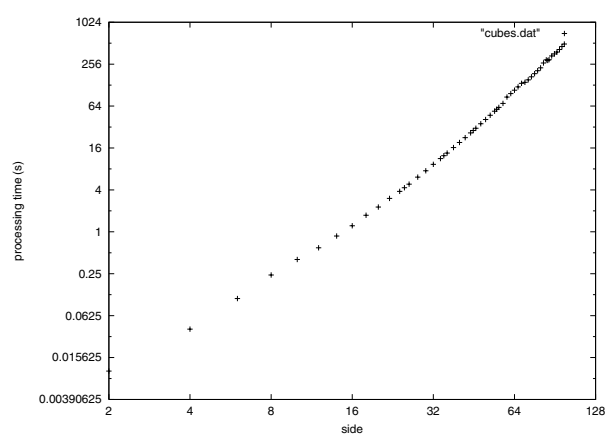

(a)

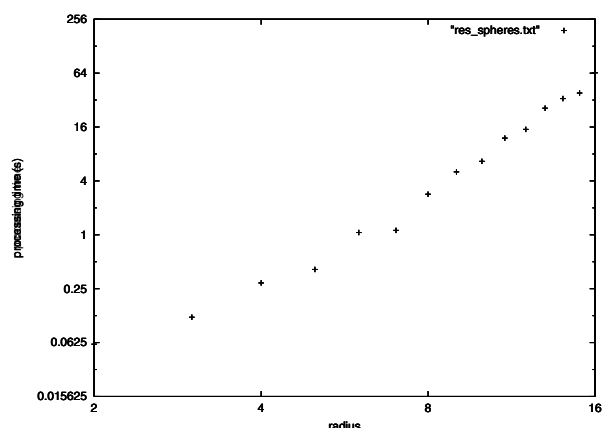

(b)

Fig. 10. Performance results: (a) for the cube; (b) for the sphere. 
which leads to an $\mathcal{O}\left(n^{8}\right)$ theoretical bound for the time complexity. We see in Figure 10 that the graph is really close to a straight line. In fact, if we consider the uncertainties due to such measurements, this result approaches very well a straight line with slope 3.5. This means that for the cube, the algorithm runs in $\mathcal{O}\left(n^{3.5}\right)$ if $n$ is the side of the square, which is quite better than the theoretical bound found in Section 5 .

We did the same job for a sphere, and the results are presented in Figure 10(b). With this object, it is harder to do a comparison with the theoretical bound: indeed, it depends on the size of the biggest plane recognized, and it is hard to find a relation between the radius of the sphere and the size of the biggest plane. Nevertheless, the number of surface voxels is in $\mathcal{O}\left(n^{2}\right)$ if $n$ is the radius of the sphere, and we can also suppose that the size of the biggest plane is a fraction of $n^{2}$. All together, we find a theoretical time complexity of $\mathcal{O}\left(n^{8}\right)$. Finally, it is interesting to notice that the curve we obtain is, as for the cube, very close to a straight line of slope 4.5. This means that the algorithm runs in $\mathcal{O}\left(n^{4.5}\right)$ which is better than the estimation of the theoretical bound.

7.2. Image Results. To finish, we give here some image results of this algorithm. For all the images presented here (Figures 11 and 12), each color corresponds to one piece of plane. For the visualization, if one surfel belongs to many pieces of planes, we display the color of the piece of plane that was recognized first.

Figure 11 presents some created and simple objects: one pyramid, a cube, a cube rotated in the grid and a chamfer cube (a cube of which one vertex has been cut by a

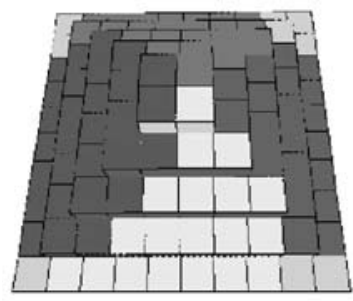

(a)

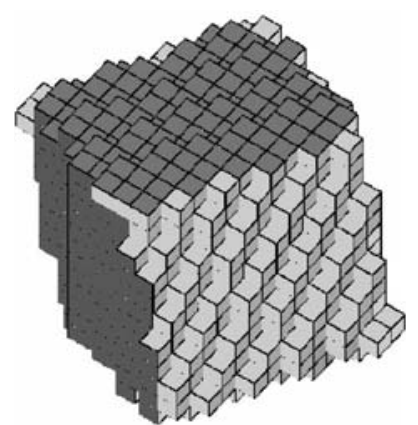

(c)

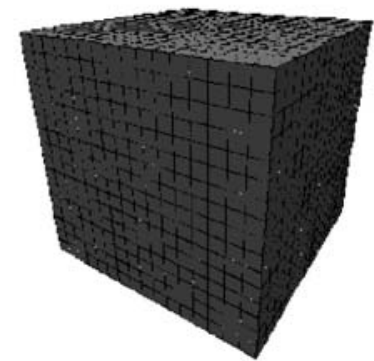

(b)

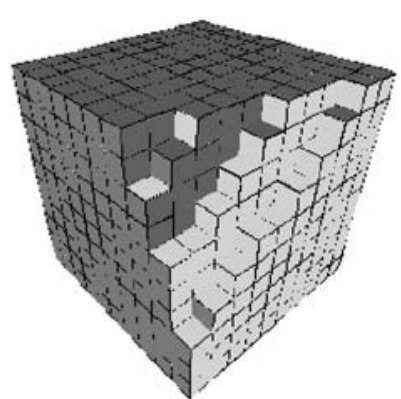

(d)

Fig. 11. Simple objects: (a) a pyramid with basis 10 and height 5; (b) a cube of side 16; (c) a cube rotated in the grid; (d) a chamfer cube. 


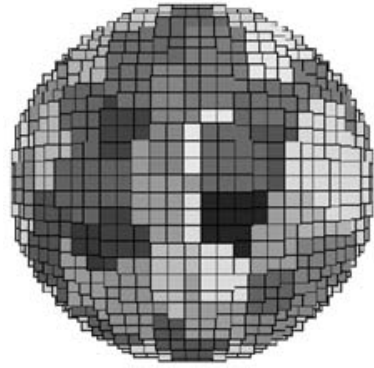

(a)

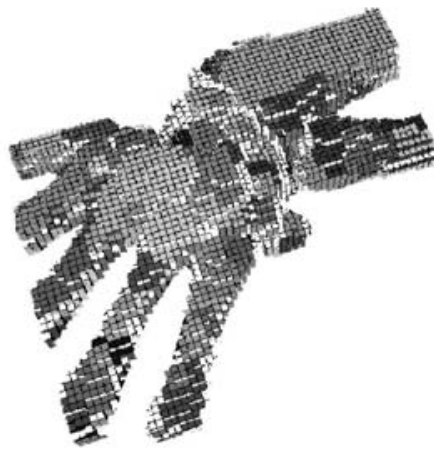

(b)

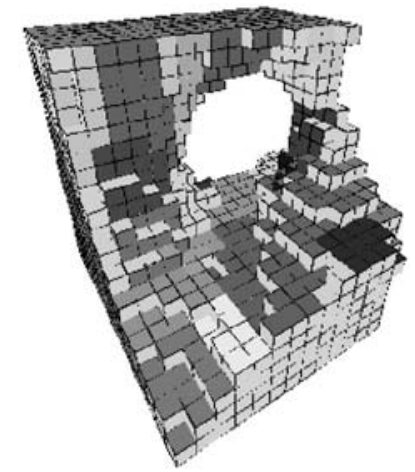

(c)

Fig. 12. (a) A sphere of radius 14; (b) a hand image; (c) a small part of a human vertebra.

plane). On the pyramid, we see that four planes have been recognized, for the four faces of the pyramid. All those planes are the same by symmetry: many voxels belong to two or more planes, and thus, with the priority rules we defined above, the plane first recognized is bigger than the others on the picture. Our algorithm recognizes the six faces of a cube for any rotation in the grid, and for a chamfer cube, it recognizes in addition the plane that cuts a vertex of this cube. As for the pyramid, the priority rules hide a big part of the sectioning plane: typically in this example, as the slopes of this plane and the face of the cube are close, the overlap between these planes is about 35 voxels.

Figure 12 gives the results for real objects: one image of a single hand's bones; one image of a piece of vertebra with high resolution. A table of the sizes of the planes recognized on the vertebra is presented in the Appendix.

8. Conclusion and Future Work. In this paper we have presented a new polynomial algorithm for the number of surface voxels to decompose the surface of any discrete volume into pieces of digital naive planes. To do so, we used an incremental naive plane recognition algorithm and we have shown some properties on the dual space associated to each piece of plane. 
Using 8-neighborhood voxels tracking, this decomposition algorithm forbids too long and narrow pieces of planes, and we analyzed some shape properties of the recognized pieces of planes. Then we analyzed the global time complexity of this algorithm, finding a polynomial bound depending on the number of surface voxels. A sharper analysis of this algorithm led us to study the influence of the different voxels tracking orders. In a last part, we made some performance tests on cubes of increasing size. These tests have shown that for the cube, practical performances are much better than the theoretical time complexity. The last images illustrated the position of the recognized pieces of plane for generated and real objects.

This work opens many future prospects, both on theoretical and practical aspects. First, some practical work can be done to improve performances: the use of a library that handles integers with arbitrary precision will enable us to run this algorithm on bigger volumes.

On the theoretical side, it would be interesting to study in more detail the structure of the dual space for a piece of plane as has been done in two-dimensional for discrete line segments [16].

Finally, this paper presented the first step of a more global goal that consists of finding a reversible polyhedrization of any discrete volume. To get such a polyhedrization, we need to transform each recognized piece of plane into a discrete polygon, a definition of which has been proposed in [17]. This supposes that we can define and place all the edges and the vertices between the found pieces of plane.

Appendix. Table of the Plane Sizes for the Vertebra (see Figure 12)

\begin{tabular}{rrrrrrrr}
\hline p.n. & Size & p.n. & Size & p.n. & Size & p.n. & Size \\
\hline 0 & 59 & 1 & 24 & 2 & 12 & 3 & 24 \\
4 & 32 & 5 & 21 & 6 & 31 & 7 & 119 \\
8 & 124 & 9 & 25 & 10 & 27 & 11 & 9 \\
12 & 15 & 13 & 12 & 14 & 18 & 15 & 9 \\
16 & 74 & 17 & 15 & 18 & 40 & 19 & 9 \\
20 & 106 & 21 & 15 & 22 & 41 & 23 & 18 \\
24 & 12 & 25 & 16 & 26 & 15 & 27 & 15 \\
28 & 9 & 29 & 9 & 30 & 16 & 31 & 23 \\
32 & 20 & 33 & 15 & 34 & 9 & 35 & 18 \\
36 & 119 & 37 & 95 & 38 & 58 & 39 & 87 \\
\hline *Plane number. & & & & & &
\end{tabular}

\section{References}

[1] W.E. Lorensen and H.E. Cline. Marching cubes: a high resolution 3d surface construction algorithm. Computer Graphics, 21(4):163-169, 1987.

[2] C.E. Kim and A. Rosenfeld. Convex digital solids. IEEE Transactions on Pattern Analysis and Machine Intelligence, PAMI-4(6):612-618, 1982.

[3] C.E. Kim and I. Stojmenović. On the recognition of digital planes in three dimensional space. Pattern Recognition Letters, 32:612-618, 1991. 
[4] Ph. Borianne and J. Françon. Reversible polyhedrization of discrete volumes. In J.-M. Chassery and A. Montanvert, editors, Discrete Geometry for Computer Imagery, pages 157-168, 1994.

[5] I. Debled-Rennesson. Etude et reconnaissance des droites et plans discrets. Ph.D. thesis, Université Louis Pasteur, Strasbourg, 1995.

[6] I. Debled-Rennesson and J.-P. Reveillès. An incremental algorithm for digital plane recognition. In Discrete Geometry for Computer Imagery, pages 207-222, 1994.

[7] L. Papier. Polyédrisation et visualisation d'objets discrets tridimensionnels. Ph.D. thesis, Université Louis Pasteur, Strasbourg, 1999.

[8] L. Papier and J. Françon. Polyhedrization of the boundary of a voxel object. In C. Bertrand, M. Couprie, and L. Perroton, editors, Discrete Geometry for Computer Imagery, number 1568 in LNCS, pages 425434. Springer-Verlag, Berlin, 1999.

[9] J. Burguet and R. Malgouyres. Strong thinning and polyhedrization of the surface of a voxel object. In G. Borgefors, I. Nyström, and G. Sanniti di Baja, editors, Discrete Geometry for Computer Imagery, number 1953 in LNCS, pages 222-234. Springer-Verlag, Berlin, 2000.

[10] J.-P. Reveillès. Géométrie discrète, calcul en nombres entiers et algorithmique. Ph.D. thesis, Université Louis Pasteur, Strasbourg, 1991.

[11] E. Andres, R. Acharya, and C. Sibata. Discrete analytical hyperplanes. Graphical Models and Image Processing, 59(5):302-309, 1997.

[12] J. Vittone and J.-M. Chassery. Recognition of digital naive planes and polyhedization. In G. Borgefors, I. Nyström, and G. Sanniti di Baja, editors, Discrete Geometry for Computer Imagery, number 1953 in LNCS, pages 296-307. Springer-Verlag, Berlin, 2000.

[13] J. Vittone. Caractérisation et reconnaissance de droites et de plans en géométrie discrète. Ph.D. thesis, Université Joseph Fourier, Grenoble, 1999.

[14] F. P. Preparata and M. I. Shamos. Computational Geometry: An Introduction. Springer-Verlag, New York, 1985.

[15] D.J. Grabiner. Farey nets and multidimensional continued fractions. Monatshefte für Mathematik, 114(1):35-61, 1992.

[16] M.D. McIlroy. A note on discrete representation of lines. AT\&T Technical Journal, 64(2):481-490, February 1984.

[17] E. Andrès. Defining discrete objects for polygonalization: the standard model. In J.-O. Lachaud, A. Braquelaire and A. Vialard, editors, Discrete Geometry for Computer Imagery, number 2301 in LNCS, pages 313-325. Springer-Verlag, Berlin, 2002. 